Elsevier required licence: (C) <2021>. This manuscript version is made available under the CC-BY-NCND 4.0 license http://creativecommons.org/licenses/by-nc-nd/4.0/

The definitive publisher version is available online at

[https://www.sciencedirect.com/science/article/pii/S0955799721001259?via\%3Dihub] 


\title{
Efficient solution of block Toeplitz systems with multiple right-hand sides arising from a periodic boundary element formulation
}

\author{
Christopher Jelich ${ }^{\mathrm{a}, *}$, Mahmoud Karimi ${ }^{\mathrm{b}}$, Nicole Kessissoglou ${ }^{\mathrm{c}}$, Steffen Marburg ${ }^{\mathrm{a}}$ \\ ${ }^{a}$ Chair of Vibroacoustics of Vehicles and Machines, Technical University of Munich, Boltzmannstrasse 15, 85748 Garching, \\ Germany \\ ${ }^{b}$ Centre for Audio, Acoustics and Vibration, University of Technology Sydney, Sydney, New South Wales, Australia \\ ${ }^{c}$ School of Mechanical and Manufacturing Engineering, The University of New South Wales, Sydney, New South Wales, Australia
}

\begin{abstract}
Block Toeplitz matrices are a special class of matrices that exhibit reduced memory requirements and a reduced complexity of matrix-vector multiplications. We herein present an efficient computational approach to solve a sequence of block Toeplitz systems arising from a block Toeplitz system with multiple right-hand sides. Two different numerical schemes are implemented for the solution of the sequence of block Toeplitz systems based on global and block variants of the generalized minimal residual (GMRES) method. The performance of the schemes is assessed in terms of the wall clock time of the iterative solution process, the number of multiplications with the block Toeplitz system matrix and the peak memory usage. To demonstrate the method, two numerical examples are presented. In the first case study, aeroacoustic prediction of an airfoil in turbulent flow is examined, which requires multiple solutions of the wall pressure field beneath the turbulent boundary layer. The fluctuating pressure on the surface of the airfoil is synthesized in terms of uncorrelated wall plane waves, whereby each realization of the wall pressure field is an input to the acoustic solver based on the boundary element method (BEM). The total acoustic response from the airfoil in turbulent flow is then obtained from an ensemble average for the number of realizations considered. The number of realizations to yield a converged solution for the wall pressure field leads to a sequence of block Toeplitz systems. The second case study examines the nonlinear eigenvalue analysis of a sonic crystal barrier composed of locally resonant C-shaped sound-hard scatterers. The periodicity of the sound barrier leads to a block Toeplitz system matrix whereas the nonlinear eigenvalue problem requires the solution of sequences of linear systems. The combined technique to solve the sequences of block Toeplitz systems using the proposed variants of the GMRES is shown to yield a computationally efficient approach for flow noise prediction and nonlinear eigenvalue analysis.
\end{abstract}

Keywords: sequence of linear systems, global Krylov solver, block Krylov solver, block Toeplitz matrix, boundary element method

\section{Introduction}

A block Toeplitz matrix is a special block matrix which contains constant blocks along each diagonal, and is one of the most well-studied classes of structured matrices [1]. A system of linear equations in

\footnotetext{
${ }^{*}$ Corresponding author

Email address: c.jelich@tum.de (Christopher Jelich)
} 
which the system matrix is a block Toeplitz matrix is called a block Toeplitz system [1,2]. Block Toeplitz systems arise in a diversity of disciplines including applied mathematics [3, 4], physical sciences and engineering [5-10], and signal processing and control [11]. Karimi et al. [12] found that applying the BEM to periodic structures leads to the formation of a block Toeplitz matrix due to the translational invariance of the free-space Green's function. The method is termed the periodic boundary element method and has been applied to problems with multi-directional periodicity [13], problems involving rotational symmetry [14] and aeroacoustic predictions of airfoils $[15,16]$. In the latter case, sequences of linear block Toeplitz systems are considered which are block Toeplitz systems with many right-hand sides. Sequences of linear block Toeplitz systems can also be found in Gaussian process modeling [17], electromagnetics [18, 19] and data analysis [20].

Direct or iterative solvers are generally employed to solve a Toeplitz system. Direct solution schemes for Toeplitz systems as well as multilevel and block Toeplitz systems have been extensively studied, for example, see [21-26]. Direct solution schemes generally exhibit a complexity of $O\left(N^{2}\right)$, with $N$ denoting the number of degrees of freedom. Recently, stable direct solvers with complexities below $O\left(N^{2}\right)$ have been proposed, for example, see [27-29] and references therein. However, the high memory requirements of direct solvers still prevent their application to large-scale problems. Iterative solvers generally require less memory than direct solvers and can be implemented without additional effort [30-32]. Employing an iterative solver for the solution of a single linear block Toeplitz system was shown to exhibit a complexity of $O\left(N \log ^{2}(N)\right)$. A prominent variant of an iterative solver is the conjugate gradient algorithm, which has been applied to symmetric positive definite Toeplitz systems employing circulant preconditioners [33, 34]. Using this algorithm, several parallelization techniques were implemented to solve multilevel Toeplitz systems [35]. Further, the minimum residual method and the generalized minimal residual method (GMRES) were used to solve indefinite and non-symmetric Toeplitz systems [36, 37]. Barrowes et al. [8] demonstrated efficient solution of an electromagnetic scattering problem involving multilevel block Toeplitz matrices using the biconjugate gradient stabilized method together with one-dimensional fast Fourier transforms.

The aforementioned iterative solution schemes belong to the class of Krylov subspace methods and can be grouped as seed/recycling, global or block Krylov subspace methods [38]. Using seed methods, a single system within the sequence of linear systems is selected and solved. Subsequently, the Krylov subspace of the seed system is reused in the solution process of the other linear systems. For $m$ linear systems, the residuals of the remaining $m-1$ linear systems are projected onto the Krylov subspace of the seed system. This often leads to a good approximation of the solution of the remaining non-seed systems [38, 39]. Seed methods can be improved further by introducing augmentation and/or deflation, which are referred to as recycling methods [40, 41]. Recycling methods have been applied to solve the Helmholtz equation discretized by the finite element method [42] as well as Maxwell's equations discretized by the boundary element method [43, 44]. In contrast, global and block methods efficiently solve all linear systems within the sequence simultaneously. As such, the solution and right-hand side vectors are concatenated into matrices. This allows matrix-matrix multiplications to be efficiently performed within the global and block iterative schemes. A further benefit of global and block methods is that they build a much larger Krylov subspace which usually yields a solution within less iterations [45, 46]. Malhotra et al. [47] applied block methods to solve exterior acoustic problems using the finite element method (FEM). Block methods have also been employed using the FEM for applications in exterior acoustics to address waveform inversion [48] and for uncertainty analysis [42].

The current work extends the capability in exterior acoustics by employing global and block iterative solvers to accelerate the solution process of a block Toeplitz system with many right-hand sides. Both global and block variants of the GMRES are employed to solve a sequence of block Toeplitz systems arising from 
a BEM formulation. Two numerical examples are considered. In the first case study, the aeroacoustic prediction of flow-induced noise from an airfoil in low Mach number turbulent flow is considered. A small segment of the airfoil was selected as a unit cell. Using a periodic BEM formulation, the acoustic problem results in a block Toeplitz system. An uncorrelated wall plane wave technique is used to synthesize the fluctuating pressures on the surface of the structure. Each realization of the synthesized wall pressure field corresponds to an input to the acoustic problem, i.e., to a right-hand side of the periodic BEM model of the airfoil. Taking a number of realizations into account results in a sequence of block Toeplitz systems. The acoustic response of each realization is predicted by solving this sequence. The final acoustic response due to flow-induced noise from the flat plate is then obtained from an ensemble average of the individual acoustic response of the realizations. In the second case study, the eigenvalues and eigenvectors of a periodic sound barrier design are analyzed. Applying the periodic BEM formulation leads to a block Toeplitz system that is implicitly frequency dependent. This yields a nonlinear eigenvalue problem which can be solved efficiently by contour integral methods [49-51]. These methods allow extraction of the eigenvalues within a predefined contour but require the solution of sequences of linear systems at multiple frequency points along the contour. For both case studies, the performance of global and block variants of the GMRES to solve the sequence of block Toeplitz systems is evaluated in terms of computation time and memory requirements. Both the global and block GMRES methods are shown to significantly reduce the overall computational effort of the solution process, yielding an efficient approach for exterior acoustic problems.

\section{Sequence of block Toeplitz systems}

A sequence of linear systems is given by

$$
\mathbf{T} \mathbf{x}^{(i)}=\mathbf{b}^{(i)}, \quad i=1, \ldots, m,
$$

where $\mathbf{T}$ is a complex-valued system matrix and $\mathbf{b}^{(i)}, \mathbf{x}^{(i)}$ respectively denote the complex-valued right-hand side vector and solution vector of the $i$-th linear system within the sequence of $m$ linear systems. When the system matrix $\mathbf{T}$ is a block Toeplitz matrix, the linear systems within eq. (1) are called block Toeplitz systems [1,2]. An $N \times N$ matrix $\mathbf{T}$ is a block Toeplitz matrix if it has constant blocks along each diagonal. Hence, a block Toeplitz matrix has the form

$$
\mathbf{T}=\left(\begin{array}{ccccc}
\mathbf{T}_{0} & \mathbf{T}_{-1} & \cdots & \cdots & \mathbf{T}_{1-s} \\
\mathbf{T}_{1} & \mathbf{T}_{0} & \mathbf{T}_{-1} & \cdots & \mathbf{T}_{2-s} \\
\vdots & \mathbf{T}_{1} & \ddots & \ddots & \vdots \\
\vdots & \vdots & \ddots & \ddots & \mathbf{T}_{-1} \\
\mathbf{T}_{s-1} & \mathbf{T}_{s-2} & \cdots & \mathbf{T}_{1} & \mathbf{T}_{0}
\end{array}\right),
$$

where each $\mathbf{T}_{j}$ is an $n \times n$ matrix. In order to solve block Toeplitz systems by means of global and block Krylov solvers, the sequence of linear systems given by eq. (1) is reformulated into

$$
\mathbf{T X}=\mathbf{B} \text {, }
$$

where the solution and right-hand side vectors are concatenated to form the solution matrix $\mathbf{X}=\left[\mathbf{x}^{(1)}, \ldots, \mathbf{x}^{(m)}\right]$ and right-hand side matrix $\mathbf{B}=\left[\mathbf{b}^{(1)}, \ldots, \mathbf{b}^{(m)}\right]$.

An efficient scheme to calculate block Toeplitz matrix products is implemented using the following procedure: (i) calculate the unique entries of the block Toeplitz matrix, (ii) construct the first block column 
of a block circulant matrix, (iii) calculate the discrete Fourier transform (DFT) of this first block column, and (iv) store the DFT of the first block column as a block diagonal matrix. The unique elements of the block Toeplitz matrix in eq. (2) are the first block row and first block column. Concatenating the unique blocks column-wise yields the following $n \times(2 s-1) n$ matrix

$$
\mathbf{Q}=\left(\begin{array}{llllllll}
\mathbf{T}_{0} & \mathbf{T}_{-1} & \cdots & \mathbf{T}_{1-s} & \mathbf{T}_{s-1} & \cdots & \mathbf{T}_{2} & \mathbf{T}_{1}
\end{array}\right) .
$$

The $\mathbf{Q}$ matrix defines the first block row of the block circulant matrix $\mathbf{C}$. A block circulant matrix is a special type of block Toeplitz matrix in which each block row is a rightward circular shift of the first block row $[2,52]$. Therefore, rightward circular shifts of $\mathbf{Q}$ form the block circulant matrix $\mathbf{C}$, i.e.

$$
\mathbf{C}=\left(\begin{array}{ccccc}
\mathbf{T}_{0} & \mathbf{T}_{-1} & \cdots & \mathbf{T}_{2} & \mathbf{T}_{1} \\
\mathbf{T}_{1} & \mathbf{T}_{0} & \mathbf{T}_{-1} & \cdots & \mathbf{T}_{2} \\
\vdots & \mathbf{T}_{1} & \ddots & \ddots & \vdots \\
\mathbf{T}_{-2} & \vdots & \ddots & \ddots & \mathbf{T}_{-1} \\
\mathbf{T}_{-1} & \mathbf{T}_{-2} & \cdots & \mathbf{T}_{1} & \mathbf{T}_{0}
\end{array}\right) .
$$

By embedding the block Toeplitz matrix into a block circulant matrix, matrix products become circular convolutions that can be quickly computed using the discrete Fourier transform (DFT) or the fast Fourier transform (FFT). A matrix product can be obtained by multiplying the block Toeplitz matrix $\mathbf{T}$ with an arbitrary matrix $\mathbf{V}$, which is equivalently expressed in terms of a block circulant matrix $\mathbf{C}$ as follows [14]

$$
\mathbf{T V}=\mathbf{Z} \quad \equiv \quad \mathbf{C} \tilde{\mathbf{V}}=\tilde{\mathbf{Z}},
$$

with $\tilde{\mathbf{V}}$ and $\tilde{\mathbf{Z}}$ being extended versions of $\mathbf{V}$ and $\mathbf{Z}$, respectively. Introducing the block DFT operator $\tilde{F}$ and its corresponding inverse operator $\mathfrak{F}^{-1}$, the block circulant matrix in eq. (6) is given by

$$
\mathbf{C}=\mathfrak{F}^{-1} \boldsymbol{\Lambda} \mathfrak{F}
$$

where $\boldsymbol{\Lambda}$ is a block diagonal matrix. This block diagonal matrix stores the block DFT of the first block column of the block circulant matrix, that is

$$
\boldsymbol{\Lambda}=\operatorname{diag}\left(\hat{\mathbf{T}}_{0}, \hat{\mathbf{T}}_{1}, \ldots, \hat{\mathbf{T}}_{-2}, \hat{\mathbf{T}}_{-1}\right),
$$

where $\left[\begin{array}{lllll}\hat{\mathbf{T}}_{0} & \hat{\mathbf{T}}_{1} & \ldots & \hat{\mathbf{T}}_{-2} & \hat{\mathbf{T}}_{-1}\end{array}\right]=\mathfrak{F}\left[\begin{array}{lllll}\mathbf{T}_{0} & \mathbf{T}_{1} & \ldots & \mathbf{T}_{-2} & \mathbf{T}_{-1}\end{array}\right]$. The forward block DFT operator is given by the Kronecker product $\otimes$ of the $(2 s-1) \times(2 s-1)$ Fourier matrix $\mathbf{F}_{2 s-1}$ and the $n \times n$ identity matrix $\mathbf{I}_{n}$, that is

$$
\mathfrak{F}=\mathbf{F}_{2 s-1} \otimes \mathbf{I}_{n} .
$$

Analogously, the inverse of the forward block DFT operator is defined by

$$
\mathfrak{F}^{-1}=\mathbf{F}_{2 s-1}^{-1} \otimes \mathbf{I}_{n} .
$$

The definition of the Fourier matrix and its inverse can be found in [1].

The extended matrices $\tilde{\mathbf{V}}$ and $\tilde{\mathbf{Z}}$ in the matrix-vector product of eq. (6) include additional unknowns. A further acceleration is achieved by only computing the necessary terms, i.e. by computing

$$
\overline{\mathbf{C}} \mathbf{V}=\mathbf{Z} \text {. }
$$


The $s n \times s n$ matrix $\overline{\mathbf{C}}$ is defined by

$$
\overline{\mathbf{C}}=\overline{\mathfrak{F}}^{-1} \boldsymbol{\Lambda} \overline{\mathfrak{F}}
$$

with the incomplete block DFT operators

$$
\overline{\mathfrak{F}}=\overline{\mathbf{F}}_{2 s-1} \otimes \mathbf{I}_{n} \quad \text { and } \quad \overline{\mathfrak{F}}^{-1}=\overline{\mathbf{F}}_{2 s-1}^{-1} \otimes \mathbf{I}_{n}
$$

The incomplete Fourier matrix $\overline{\mathbf{F}}_{2 s-1}$ contains the first $s$ columns of $\mathbf{F}_{2 s-1}$, whereas the incomplete inverse Fourier matrix $\overline{\mathbf{F}}_{2 s-1}^{-1}$ contains the first $s$ rows of $\mathbf{F}_{2 s-1}^{-1}$.

\section{Krylov subspace solvers}

\subsection{Block Krylov subspace solver}

Block Krylov subspace solvers can be derived from standard Krylov subspace solvers by modifying the way the Krylov subspace is built. Block Krylov methods generate an approximate solution $\mathbf{X}_{k} \in \mathbb{C}^{N \times m}$ in each iteration $k$ such that the following relation holds

$$
\mathbf{X}_{k}-\mathbf{X}_{0} \in \mathcal{K}_{k}^{\square}\left(\mathbf{T}, \mathbf{R}_{0}\right) .
$$

Here, $\mathbf{X}_{0} \in \mathbb{C}^{N \times m}$ is an arbitrary initial estimate of the solution matrix, $\mathbf{R}_{0}=\mathbf{B}-\mathbf{T} \mathbf{X}_{0}$ is the initial residual and $\mathcal{K}_{k}^{\square}\left(\mathbf{T}, \mathbf{R}_{0}\right)$ is the block Krylov subspace of iteration $k$. The superscript ( $)^{\square}$ denotes the block nature of the subspace and is given by [38]

$$
\mathcal{K}_{k}^{\square}\left(\mathbf{T}, \mathbf{R}_{0}\right)=\left\{\sum_{i=0}^{k-1} \mathbf{T}^{i} \mathbf{R}_{0} \boldsymbol{\gamma}_{i} ; \boldsymbol{\gamma}_{i} \in \mathbb{C}^{m \times m}\right\} .
$$

Equation (15) shows that every column of the matrix $\mathbf{X}_{k}-\mathbf{X}_{0}$ is approximated by a linear combination of every column of the matrices spanning the block Krylov subspace $\mathcal{K}_{k}^{\square}\left(\mathbf{T}, \mathbf{R}_{0}\right)$. Compared to standard Krylov subspace solvers, the block Krylov subspace is of much larger size. Each iteration adds up to $m$ additional Krylov vectors to the subspace instead of just a single vector in the case of standard Krylov solvers. As such, a greater number of Krylov vectors is available for the approximation of the solution in each iteration and convergence is generally reached in less iterations [38]. However, the computational efficiency comes at the cost of additional memory requirements. Further, linearly or nearly linearly dependent columns in the block Krylov subspace lead to a breakdown of the solver. Deflation techniques which detect and delete linearly dependent columns prevent this breakdown [38]. In addition, linearly dependent right-hand side vectors can be detected upfront. An elegant implementation was introduced by Langou [43], comprising the performance of a singular value decomposition (SVD) of the right-hand side matrix of eq. (3) and truncating the SVD at a prescribed tolerance. This leads to a dimension reduction of the right-hand side matrix, thus reducing the computational effort.

The block variant of the GMRES introduced by Vital [53] is herein employed. The block GMRES uses a block Arnoldi process to build the block-orthogonal basis of $\mathcal{K}_{k}^{\square}\left(\mathbf{T}, \mathbf{R}_{0}\right)$. Similar to the standard GMRES, this orthogonal basis is stored and extended within every iteration. As such, the block GMRES requires a significant amount of memory which increases linearly with $k$. Remedies to the large memory requirements are restarting the algorithm [54] and applying preconditioning techniques [48, 55]. 


\subsection{Global Krylov subspace solver}

Global Krylov subspace solvers generate an approximate solution $\mathbf{X}_{k}$ in each iteration $k$ such that the following relation holds

$$
\mathbf{X}_{k}-\mathbf{X}_{0} \in \mathcal{K}_{k}\left(\mathbf{T}, \mathbf{R}_{0}\right)
$$

In contrast to block methods, global methods rely on a matrix Krylov subspace $\mathcal{K}_{k}\left(\mathbf{T}, \mathbf{R}_{0}\right)$ that is extended in each iteration $k$ as follows

$$
\mathcal{K}_{k}\left(\mathbf{T}, \mathbf{R}_{0}\right)=\left\{\sum_{i=0}^{k-1} \mathbf{T}^{i} \mathbf{R}_{0} \gamma_{i} ; \gamma_{i} \in \mathbb{C}\right\} .
$$

Using the global Krylov subspace, the entire matrix $\mathbf{X}_{k}-\mathbf{X}_{0}$ is approximated by a linear combination of the matrices which span the matrix Krylov subspace. This clearly differs from block methods which rely on the block Krylov subspace in eq. (15) and approximate each column of $\mathbf{X}_{k}-\mathbf{X}_{0}$ independently. Consequently, the search space of global methods is smaller than the search space of block methods, yielding slower convergence [38].

The global variant of the GMRES method introduced by Jbilou et al. [56] employs the global Arnoldi algorithm. This algorithm creates $F$-orthonormal matrices $\mathbf{V}_{i}$, i.e. $\operatorname{tr}\left(\mathbf{V}_{i}^{*} \mathbf{V}_{j}\right)=0$ for $i \neq j$ and $i, j=0, \ldots, k-1$ in its $k$-th iteration. Here, $\operatorname{tr}()$ denotes the trace and ()$^{*}$ denotes the conjugate transpose. Linear dependence of the matrices spanning the Krylov subspace does not occur. However, linear dependent right-hand sides can be handled following the aforementioned approach of Vital [53].

\section{Numerical examples}

\subsection{Aeroacoustic prediction of an airfoil in turbulent flow}

In the first numerical example, aeroacoustic prediction of an airfoil in turbulent flow is examined. An incompressible flow passes a flat plate with a Reynolds number based on chord $R e_{c}=4.9 \cdot 10^{5}$ and Mach number $M=0.1$. At this Reynolds number, the flow is in a turbulent unsteady regime. The plate has a chord length of $200 \mathrm{~mm}$, a variable span $l$ and a thickness of $5 \mathrm{~mm}$, as shown in fig. 1. The leading edge is circular with a diameter of $5 \mathrm{~mm}$ while the trailing edge is a symmetric wedge shape with an apex angle of $12^{\circ}$.

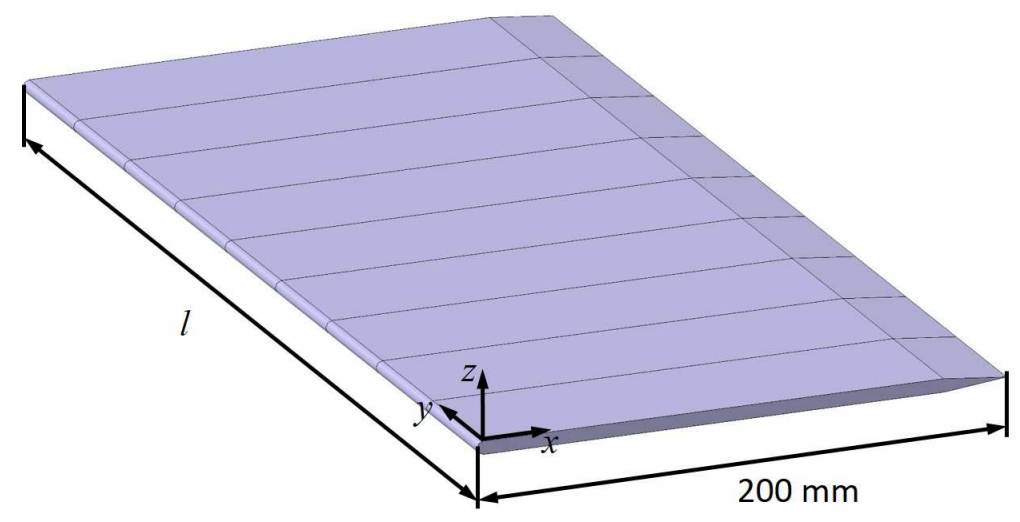

Figure 1: Flat plate airfoil divided into small segments in the spanwise direction. The local coordinates in the streamwise and spanwise directions are denoted by $x$ and $y$ respectively. 
Assuming a time-harmonic dependence of the form $\mathrm{e}^{-\mathrm{i} \omega t}$, where $\mathrm{i}=\sqrt{-1}, \omega=2 \pi f$ is the circular frequency, $f$ is the frequency in $\mathrm{Hz}$ and $t$ is time, the Helmholtz equation is given by

$$
\Delta p(\mathbf{x}, \omega)+k_{\mathrm{a}}^{2} p(\mathbf{x}, \omega)=-q(\mathbf{x}, \omega) .
$$

$p(\mathbf{x}, \omega)$ is the acoustic pressure at point $\mathbf{x}, \Delta$ is the Laplacian operator, $k_{\mathrm{a}}=\omega / c$ is the acoustic wave number, $c$ is the speed of sound and $q$ is the source term. The plate is divided into segments in the spanwise direction as shown in fig. 1, where each segment corresponds to a unit cell. Applying the boundary element method to the flat plate, the following block Toeplitz system is obtained [12, 13]

$$
\mathbf{T} \mathbf{p}=\mathbf{p}_{\text {inc }},
$$

where $\mathbf{T}$ is a complex-valued, non-symmetric block Toeplitz matrix in the form of eq. (2). Each block within the block Toeplitz matrix is of dimension $n \times n$, with $n$ denoting the number of degrees of freedom of the unit cell. Considering $s$ plate segments, $\mathbf{T}$ is of dimension $N \times N$ with $N=n s$ denoting the total number of degrees of freedom. In eq. (19), $\mathbf{p}_{\text {inc }}$ and $\mathbf{p}$ represent the incident acoustic pressure and response acoustic pressure at discrete nodal points on the boundary of the flat plate. In contrast to the underlying geometry, the incident and response pressures do not need to fulfill periodicity.

The incident acoustic pressure originates from the pressure beneath a turbulent boundary layer arising from turbulent flow around the plate. This incident pressure is called the wall pressure field (WPF) and can be calculated using the uncorrelated wall plane wave (UWPW) technique [57, 58]. Different realizations of the random WPF are generated, where each realization is an input to an acoustic solver based on the BEM. The WPF for the $l$-th realization can be represented by a set of UWPWs at a node of the boundary element mesh as follows $[16,57]$

$$
p_{\text {inc }}^{(l)}(\mathbf{x}, \omega)=\sum_{i=1}^{N_{x}} \sum_{j=1}^{N_{y}} \sqrt{\frac{\phi_{p p}\left(k_{x}, k_{y}, \omega\right) \delta k_{x} \delta k_{y}}{4 \pi^{2}}} \mathrm{e}^{\mathrm{i}\left(k_{x} x+k_{y} y+\varphi_{i j}^{(l)}\right)},
$$

where $\delta k_{x}$ and $\delta k_{y}$ are the wavenumber resolutions in streamwise and spanwise directions of the wavenumber space, respectively, and $N_{x}, N_{y}$ are the number of points considered along the $k_{x}$ and $k_{y}$ directions, respectively. The phase $\varphi$ is sampled from a uniform random distribution within $[0,2 \pi]$. The cross spectral density of the WPF denoted by $\phi_{p p}$ can be expressed in terms of the auto spectral density $\Psi_{p p}$ of the WPF and the normalized cross spectral density $\tilde{\phi}_{p p}$ of the WPF as follows $[57,59]$

$$
\phi_{p p}\left(k_{x}, k_{y}, \omega\right)=\Psi_{p p}(\omega)\left(\frac{U_{c}}{\omega}\right)^{2} \tilde{\phi}_{p p}\left(k_{x}, k_{y}, \omega\right),
$$

where $U_{c}$ denotes the convective velocity. Using eqs. (20) and (21), a deterministic acoustic load is calculated for each realization.

Taking a total of $m$ realizations into account, the block Toeplitz system of eq. (19) is reformulated into a sequence of block Toeplitz systems as

$$
\mathbf{T P}=\mathbf{P}_{\text {inc }},
$$

where the matrix $\mathbf{P}=\left[\mathbf{p}^{(1)}, \ldots, \mathbf{p}^{(m)}\right]$ contains the acoustic pressure of each realization and the right-hand side matrix $\mathbf{P}_{\text {inc }}=\left[\mathbf{p}_{\text {inc }}^{(1)}, \ldots, \mathbf{p}_{\text {inc }}^{(m)}\right]$ contains the incident pressure of each realization. Solving the sequence in eq. (22) corresponds to calculating the acoustic pressure on the boundary of the flat plate for each realization. The far-field acoustic pressure $\mathbf{p}_{\mathrm{f}}=\left[p_{\mathrm{f}}^{(1)}, \ldots, p_{\mathrm{f}}^{(m)}\right]$ at a single field point $\mathbf{x}_{\mathrm{f}}$ for each realization is then computed by

$$
\mathbf{p}_{\mathrm{f}}=-\mathbf{h}_{\mathrm{f}} \mathbf{P} \text {, }
$$


where $\mathbf{h}_{\mathrm{f}}$ is the far-field boundary element matrix as defined in [60]. Finally, the scattered acoustic response at each frequency is obtained from an ensemble average of the far-field acoustic pressure of all realizations as follows

$$
S_{p p}(\mathbf{x}, \omega)=\mathbf{E}\left[p_{\mathrm{f}}^{(l)}(\mathbf{x}, \omega)\left(p_{\mathrm{f}}^{(l)}(\mathbf{x}, \omega)\right)^{*}\right]_{l \in\{1, \ldots, m\}},
$$

where $\mathbf{E}[$ ] represents the ensemble average over the number of realizations.

\subsection{Eigenvalue analysis of a locally resonant sonic crystal barrier}

Sonic crystals are periodic arrangements of sound scatterers, and have been receiving growing interest in their potential for use as a noise barrier [61, 62]. Significant sound attenuation in the shadow zone of a sonic crystal barrier is shown to occur in broad and narrow frequency bands respectively attributed to the periodicity and local resonance of the scatterers $[63,64]$. The second numerical example considers a finite sonic crystal composed of four C-shaped cylindrical shells as shown in fig. 2. Each cylinder corresponds to a periodic segment and has a height of $1 \mathrm{~m}$, an outer radius of $0.1 \mathrm{~m}$, inner radius of $0.05 \mathrm{~m}$ and a slit width of $0.05 \mathrm{~m}$. The ground is assumed to be sound-hard and the distance between the centers of neighboring C-shaped cylinders is $0.3 \mathrm{~m}$.

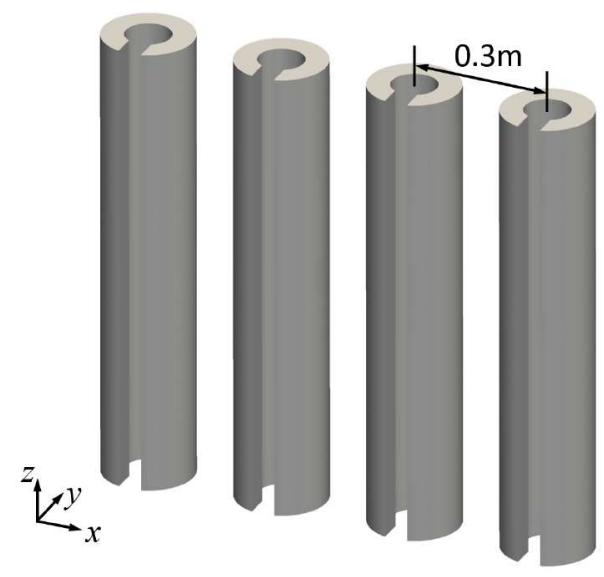

Figure 2: Sonic crystal barrier design with four C-shaped cylinders.

Applying the periodic boundary element method yields a block Toeplitz system similar to eq. (22) with an $N \times N$ block Toeplitz matrix $\mathbf{T}$ that has constant $n \times n$ matrix blocks along its diagonal. Setting the right-hand side to zero yields the following nonlinear eigenvalue problem

$$
\mathbf{T}(\tilde{\omega}) \mathbf{\Phi}=\mathbf{0},
$$

with the complex eigenvalue parameter $\tilde{\omega}$ and the nonzero eigenvector $\boldsymbol{\Phi}$. The real part of $\tilde{\omega}$ corresponds to the eigenfrequency whereas the imaginary part describes the damping of the corresponding mode. The eigenfrequencies and modal damping of the periodic structure are of interest since sound attenuation occurs around the eigenfrequencies and the mode shapes provide valuable insight on the effects leading to the attenuation. For infinite periodic arrangements, an eigenvalue analysis of a single unit cell is sufficient [65]. However, for small periodic arrangements, the entire geometry needs to be considered [66].

Contour integral methods are an efficient measure to solve the nonlinear eigenvalue problem in eq. (25). All eigenvalues and corresponding eigenvectors are extracted within a predefined contour in the complex 
plane, as shown in fig. 3. A specific contour integral method is the block Sakurai Sugiura (block-SS) method [49]. It involves transforming the nonlinear eigenvalue problem into the smaller generalized eigenvalue problem given by

$$
\left(\mathbf{H}_{2}-\hat{\omega} \mathbf{H}_{1}\right) \hat{\mathbf{\Phi}}=\mathbf{0},
$$

with the $K L \times K L$ Hankel matrices $\mathbf{H}_{1}$ and $\mathbf{H}_{2}$, where $K$ and $L$ denote parameters of the eigenvalue solver. The solution of eq. (26) yields the complex eigenvalue parameters $\hat{\omega}$ which coincide with those eigenvalue parameters $\tilde{\omega}$ of the original nonlinear eigenvalue problem that lie inside the contour $C$. The Hankel matrices are formed by

$$
\mathbf{H}_{\mathbf{1}}=\left[\mathbf{M}_{i+j-2}\right]_{i, j=1}^{K} \quad \text { and } \quad \mathbf{H}_{\mathbf{2}}=\left[\mathbf{M}_{i+j-1}\right]_{i, j=1}^{K},
$$

based on the positive integer $K$ and the sequence of moments

$$
\mathbf{M}_{k}=\frac{1}{2 \pi \mathrm{i}} \oint_{\mathcal{C}} z^{k} \mathbf{V}^{*} \mathbf{T}(z)^{-1} \mathbf{V} \mathrm{d} z, \quad k=0,1, \ldots, 2 K-1,
$$

of size $L \times L$. Calculating the moments involves an integration along the contour $C$ with complex coordinate $z$ and a random $n \times L$ matrix $\mathbf{V}$ with its conjugate transpose denoted by $\mathbf{V}^{*}$. Integrating eq. (28) with the trapezoidal rule along a circular contour yields

$$
\mathbf{M}_{k} \approx \frac{1}{N_{\mathrm{c}}} \sum_{j=0}^{N_{\mathrm{c}}-1} \rho\left(\frac{z_{j}-\gamma}{\rho}\right)^{k+1} \mathbf{V}^{*} \mathbf{T}\left(z_{j}\right)^{-1} \mathbf{V},
$$

where $z_{j}=\gamma+\rho \mathrm{e}^{\mathrm{i} \theta_{j}}$ is one of the $N_{\mathrm{c}}$ contour points [49]. The complex value of $z_{j}$ is given in polar coordinates in terms of angle $\theta_{j}$, center $\gamma$ and radius $\rho$ of the circular contour. Once the small eigenvalue problem given by eq. (26) is set up and solved, the eigenvalue parameters $\hat{\omega}$ and eigenvectors $\hat{\Phi}$ can be projected back to the original problem. The reader is referred to $[49,67]$ for details on the solution techniques for eq. (26) and the back transformation.

The accuracy of the eigenvalue solver is mainly determined by the number of contour points $N_{\mathrm{c}}$ and the value of $L$. A large value of $N_{\mathrm{c}}$ leads to a more accurate approximation of eq. (28) whereas a large value of $L$ results in a larger subspace, i.e. a larger generalized eigenvalue problem given by eq. (26). The calculation of $\mathbf{T}\left(z_{j}\right)^{-1} \mathbf{V}$ in eq. (29) is performed once for each of the $N_{\mathrm{c}}$ contour points and involves the $n \times L$ matrix $\mathbf{V}$ as the right-hand side. Hence, highly accurate eigenvalue estimates require the solution of a large number of linear systems with many right-hand sides. A sophisticated choice of the iterative solver to calculate $\mathbf{T}\left(z_{j}\right)^{-1} \mathbf{V}$ has great potential to reduce the overall computational cost of the nonlinear eigenvalue solver. Note that the structure of eq. (29) is not unique to the block-SS method but also occurs in other contour integral methods such as the block-SS with resolvent sampling [68] or nonlinear FEAST algorithms [51].

\section{Numerical results}

\subsection{Aeroacoustic prediction of an airfoil in turbulent flow}

Both block and global variants of the GMRES are applied to solve the sequence of block Toeplitz systems defined in eq. (22), arising from the prediction of the aeroacoustic response of a flat plate excited by turbulent flow using the periodic boundary element formulation in conjunction with the UWPW technique. In this example, each periodic segment of the plate sketched in fig. 1 is discretized using 376 linear discontinuous boundary elements. This yields a total of $n=1504$ degrees of freedom per segment. Four different 


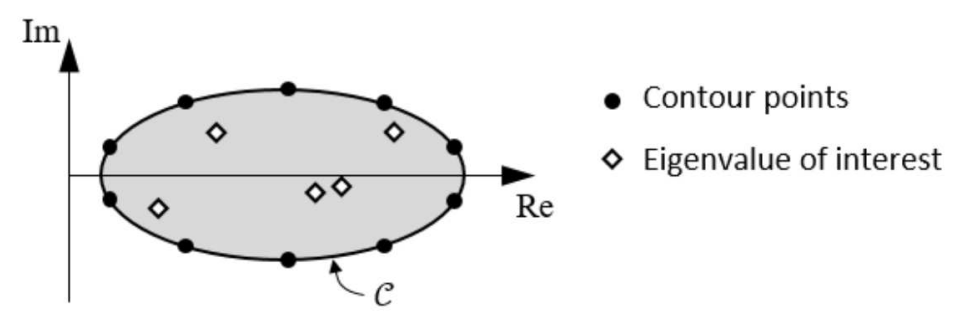

Figure 3: Contour points $z_{j}$ on the contour $C$ that encloses all eigenvalues of interest.

cases are studied in which the span length $l$ of the flat plate is varied by varying the number of segments $s$. Here, 25, 50, 75 and 100 segments are considered, corresponding to numerical models with a total of 37600 , 75200,112800 and 150400 degrees of freedom, respectively. The analyses were performed at a frequency of $f=2600 \mathrm{~Hz}$ unless specified otherwise. The plate is in air with sound speed of $c=340 \mathrm{~m} / \mathrm{s}$.

A total of $m=30$ realizations need to be taken into account to achieve an average error of the scattered acoustic pressure of less than $1 \mathrm{~dB}[16,57]$. The solution of the acoustic system with 60 and 90 realizations has also been considered in order to investigate the complexity of the linear solvers with respect to different numbers of right-hand sides. The performances of both block GMRES and global GMRES are compared to the performance of the standard GMRES algorithm by examining the total time to solution, the number of equivalent matrix-vector products and the required memory. The global and block GMRES solve the full sequence of block Toeplitz systems of eq. (22) whereas the standard GMRES solves each of the $m$ block Toeplitz systems independently. The accuracy of the solution of each block Toeplitz system is tracked by computing the norm of the relative residual in each iteration. For the global GMRES, the Frobenius norm of the residual matrix $\mathbf{R}$ is calculated, whereas for the GMRES and block GMRES, the $l^{2}$-norm of the residual of each individual block Toeplitz system is calculated. Convergence is achieved whenever a relative tolerance of $10^{-8}$ is met for all systems. Preliminary studies showed that for the case study presented here, linear dependence of the basis vectors does not occur within the solution process. Therefore, deflation techniques are not included. Furthermore, no restarting and no preconditioning is applied. The calculations were performed using MATLAB R2018b on a desktop PC with $128 \mathrm{~GB}$ of RAM and 6 physical cores running at $3.5 \mathrm{GHz}$.

\subsubsection{Acoustic response}

The acoustic response of the flat plate airfoil subject to incompressible flow was calculated using eq. (24) at 400 field points located on a circle circumscribing the flat plate. The circle has a radius of $585 \mathrm{~mm}$, lies in the $x$-z-plane and is centered at $(x, y, z)=(100 \mathrm{~mm}, l / 2,-2.5 \mathrm{~mm})$. Figure 4 shows the directivity of the scattered field at frequencies of $2600 \mathrm{~Hz}, 4200 \mathrm{~Hz}$ and $6000 \mathrm{~Hz}$ for a plate with span length $l=150 \mathrm{~mm}$. Only a limited amount of noise is radiated in the downstream and upstream direction of the flow which corresponds to $0^{\circ}$ and $180^{\circ}$, respectively. The absolute pressure is symmetric with respect to the $x-y$-plane and the phase of the acoustic waves on the top side are shifted by half a wavelength with respect to the acoustic waves on the bottom side. The noise that propagates in the upstream direction is reduced due to destructive interference of the acoustic waves which are diffracted at the leading edge. Further, acoustic waves which are scattered by the trailing edge are backscattered by the leading edge and cause multiple lobes in the directivity plot. The complexity of the acoustic directivity increases with increasing frequency as indicated by the increasing number of lobes, with a corresponding decrease in amplitude. 


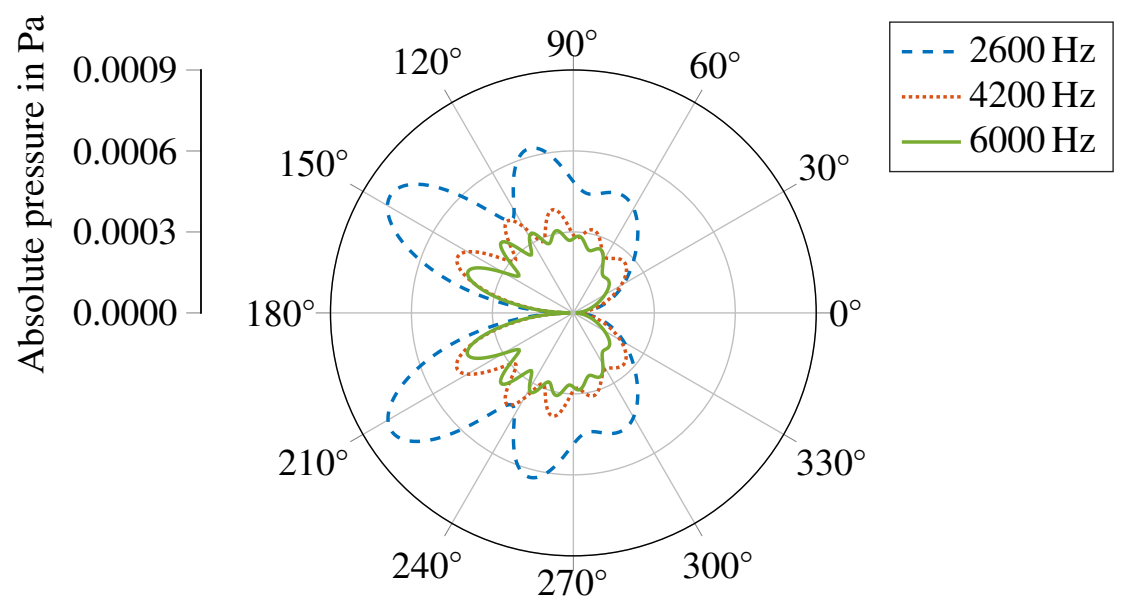

Figure 4: Directivity of the scattered absolute acoustic pressure (in Pascal) on a circle with radius $585 \mathrm{~mm}$ located in the $x$-z-plane with its center at $(x, y, z)=(100 \mathrm{~mm}, 75 \mathrm{~mm},-2.5 \mathrm{~mm})$, for a plate with span length $l=150 \mathrm{~mm}$. The downstream and upstream directions of the flow correspond to $0^{\circ}$ and $180^{\circ}$, respectively.

\subsubsection{Computational effort}

The performances of all three Krylov solvers are initially investigated by comparing the time of the iterative solution processes. Figure 5 presents the wall clock time as a function of the considered number of right-hand sides for a plate comprising 75 segments, corresponding to 112800 degrees of freedom. The solution times for all three variants of GMRES scale linearly with the number of right-hand sides. The block GMRES algorithm is the fastest in each case followed by the global GMRES algorithm, and both are significantly faster than standard GMRES. A beneficial deviation from linear scaling in time is observed for the block variant attributed to the varying number of iterations until convergence is reached. To solve the block Toeplitz systems with 30,60 and 90 right-hand sides, the block GMRES performs 10, 9 and 8 iterations, respectively. The size of the block Krylov subspace increases with an increased number of right-hand sides and convergence is reached in less iterations compared to the other Krylov solvers. This capability is unique to block Krylov subspace methods. In contrast, the global GMRES performed 12 iterations to solve the full sequence of block Toeplitz systems, and the GMRES performed 360 iterations to solve the block Toeplitz systems, independent of the number of right-hand sides.

For all three solvers, the convergence behavior of the solution of the plate model with 112800 degrees of freedom and 30 right-hand sides is presented in fig. 6. Multiplications of the system matrix are expressed in terms of equivalent matrix-vector products, i.e., multiplying the $N \times N$ system matrix with an $N \times m$ matrix corresponds to $m$ equivalent matrix-vector products. The standard GMRES solves each of the 30 block Toeplitz systems consecutively, resulting in a total of 360 matrix-vector products. The convergence plot resembles a sawtooth profile, where each tooth represents the convergence of the solution of a single system. As such, using the standard GMRES, the solution of each block Toeplitz system is equally expensive in terms of matrix multiplications. The global and block GMRES solve all block Toeplitz systems simultaneously, yielding a single monotonically decreasing curve for both cases. A total of 12 iterations are required in the case of the global GMRES whereas only 10 iterations of the block GMRES are performed until convergence is reached. This corresponds to 360 and 300 equivalent matrix-vector products, respectively.

Solving a linear system in less iterations usually comes at the cost of additional computational effort in other parts of the solution process. In the present case, the duration of each iteration can be separated 


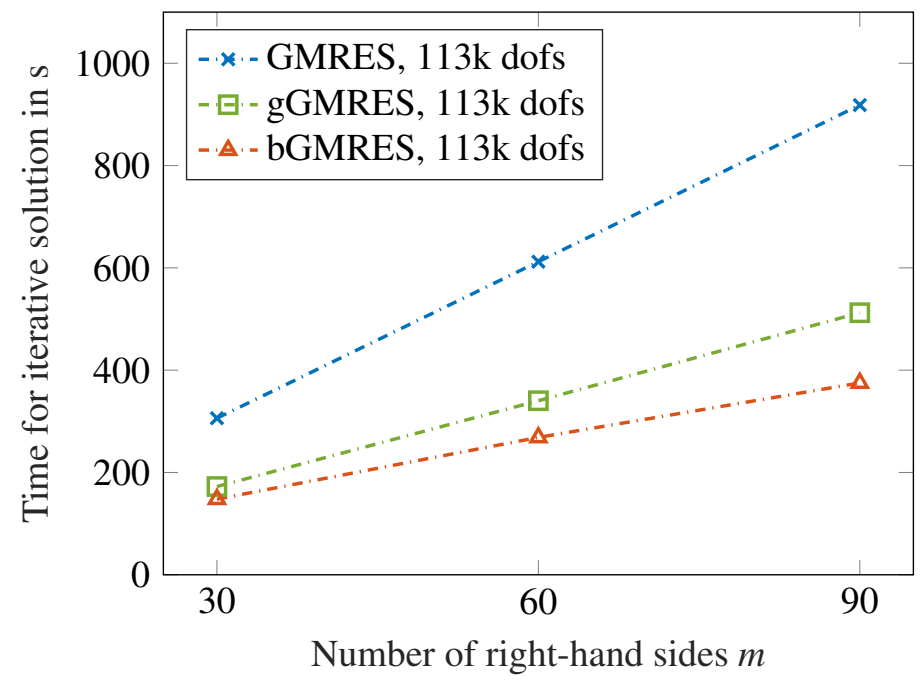

Figure 5: Wall clock time (in seconds) for solving the sequence of block Toeplitz systems with 112800 degrees of freedom (dofs) using GMRES, global GMRES (gGMRES) and block GMRES (bGMRES) for 30, 60 and 90 right-hand sides.

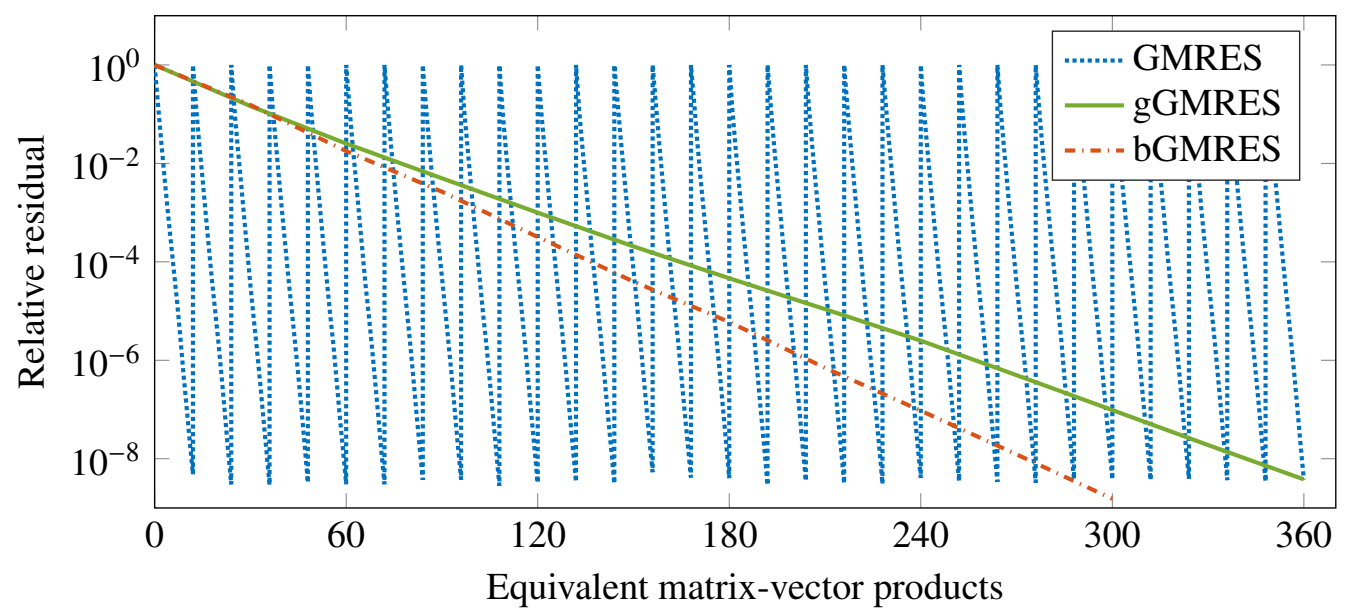

Figure 6: Convergence of the relative residual of the solutions calculated using standard GMRES, global GMRES (gGMRES) and block GMRES (bGMRES) for a plate model with 112800 degrees of freedom and 30 right-hand sides. Multiplications of the system matrix with another matrix are expressed in terms of equivalent matrix-vector products. 




Figure 7: Wall clock time (in seconds) for solving the sequence of block Toeplitz systems with 112800 degrees of freedom and 90 right-hand sides using standard GMRES, global GMRES (gGMRES) and block GMRES (bGMRES). The total time is separated into time spent for system matrix multiplication, orthogonalizing the new basis and solving a minimization problem.

into three parts: system matrix multiplication, orthogonalization of the new basis and solution of a minimization problem. Figure 7 compares the time each solver spends on these operations while solving the block Toeplitz system of a plate model with 112800 degrees of freedom and 90 right-hand sides. The first step involving multiplication of the system matrix is the most costly operation, which respectively takes up to $98.7 \%, 96.6 \%$ and $90.3 \%$ of the total solution time for the GMRES, global GMRES and block GMRES. For the case of 90 right-hand sides, GMRES and global GMRES perform a total of 1080 equivalent matrixvector products. Although both solvers require the same amount of equivalent matrix-vector products, the time both solvers spend performing these products differs by a factor of 1.81 . This is mainly related to the benefit of performing matrix-matrix multiplications in the global GMRES instead of matrix-vector multiplications in GMRES. This benefit also occurs within the block GMRES, where 8 iterations are performed until convergence is reached which corresponds to 720 equivalent matrix-vector products. An additional reduction in computational time is achieved since the block GMRES requires less iterations in total, which accounts for the difference in the solution time of the global and block variants. The aforementioned additional computational effort arises in orthogonalizing the new basis and solving the minimization problem. The global GMRES requires slightly more time during the orthogonalization process. In the block GMRES, more time is spent solving the minimization problem. However, the additional effort is only minor since the matrix multiplications take up the largest fraction of the solution time.

Finally, the solution time for different widths of the plate is investigated. Different values of the span length $l$ are obtained by modifying the number of segments $s$. The number of segments selected here are 25, 50, 75 and 100 corresponding to a total of 37600, 75200, 112800 and 150400 degrees of freedom. In fig. 8, the solution time is compared for both block and global GMRES considering all four plate models. The solution time scales linear for both variants with the aforementioned deviations occurring for block GMRES. Applying global GMRES leads to a higher wall clock time in all four cases. The average speedup of the block variant compared to the global variant is about 1.31. Compared to the standard GMRES, the average speedup is 2.34 . In fig. 8, a tendency towards higher speedup rates for block Toeplitz systems with increasing number of right-hand sides is observed. This especially holds for the plate model with 150400 degrees of freedom where the maximum speedup rates of 1.42 with respect to global GMRES and 2.53 with respect to standard GMRES are observed. 


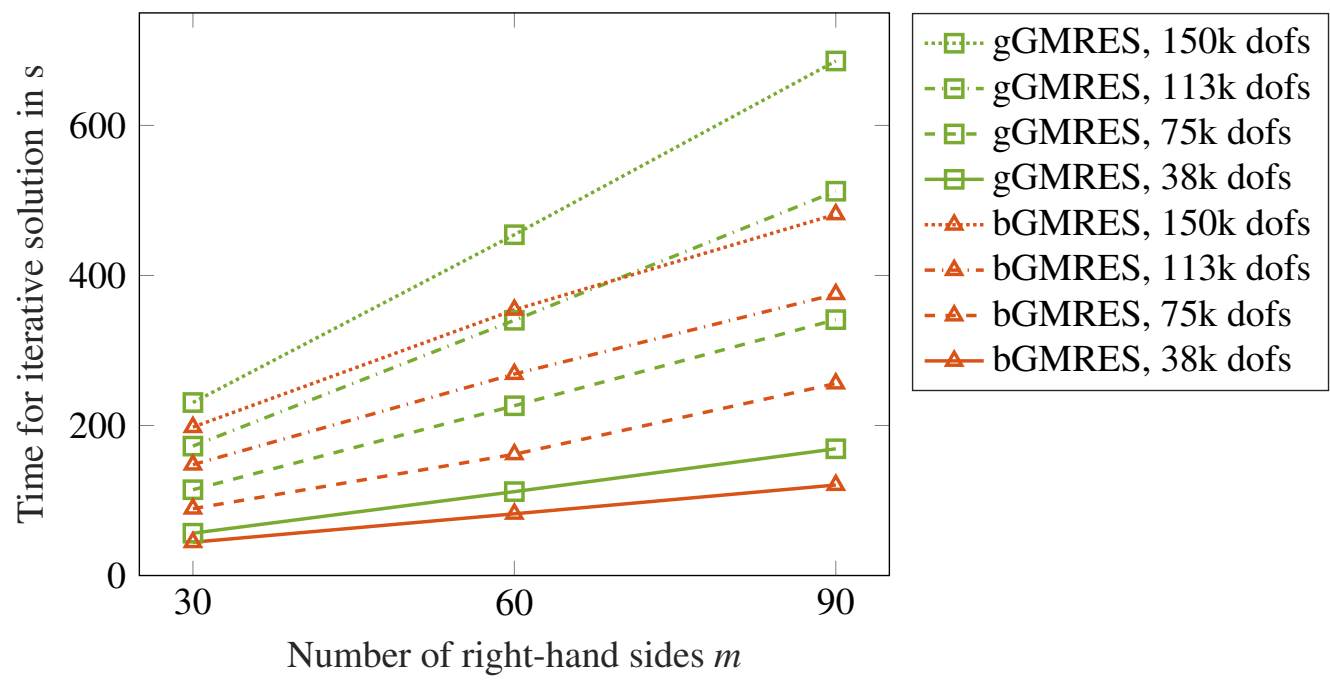

Figure 8: Wall clock time (in seconds) for solving the sequence of block Toeplitz systems using global GMRES and block GMRES taking 30, 60 and 90 right-hand sides and plate models with 37600, 75200, 112800 and 150400 degrees of freedom (dofs).

\subsubsection{Memory requirements}

The memory requirements of all three solvers is herein examined. The peak memory usage is shown in fig. 9. Since standard GMRES solves each linear system within the sequence subsequently, the memory usage is independent of the number of right-hand sides, scaling $O(N(k+5))$ with the number of iterations $k$. In the case of 150400 degrees of freedom, the standard GMRES uses a total of $36 \mathrm{MB}$ of memory. In contrast, the memory requirements of the global and block variants scale $O(N m(k+5))$ and therefore exhibit linear scaling with both the number of degrees of freedom $N$ and the number of right-hand sides $m$. The deviation from linear scaling in fig. 9 is due to the varying number of iterations until convergence is reached using block GMRES. For the solution of the numerical case study considered in this work, a standard desktop computer is adequate for the memory requirement. For problems exhibiting slower convergence or higher numbers of degrees of freedom, the memory requirements may become impracticable. To address this issue, restarting the solver as well as preconditioning can be employed.

\subsection{Eigenvalue analysis of a locally resonant sonic crystal barrier}

The second case study is an eigenvalue analysis of a periodic sound barrier design. Applying the periodic boundary element method leads to a nonlinear eigenvalue problem which can be solved by contour integral methods. In this work, the nonlinear eigenvalue solver block-SS is employed [49]. The scheme requires the solution of $N_{\mathrm{c}}$ linear systems with $L$ right-hand sides along a predefined contour $C$ according to eq. (29). Both block and global variants of GMRES are applied to these sequences of linear equations to accelerate the eigenvalue computations. The sonic crystal barrier design comprising four $\mathrm{C}$-shaped cylinders as shown in fig. 2 is examined. Each cylinder is discretized with 2800 boundary elements featuring 11200 pressure degrees of freedom. This leads to a total of 44800 pressure degrees of freedom. The design of fig. 2 features resonances at the Helmholtz frequency of the C-shaped cross section which is estimated at $382.0 \mathrm{~Hz}$ based on a two-dimensional finite element analysis of a single $\mathrm{C}$-shaped cross section. The circular contour of the eigenvalue solver has a center point at $405 \mathrm{~Hz}$ and a radius of $75 \mathrm{~Hz}$ and as such, encloses the estimated Helmholtz frequency. The contour is discretized with $N_{\mathrm{c}}=16$ contour points shown in fig. 10 and a value of $K=4$ is chosen. Six different runs of the block-SS are compared where the value of $L$ is varied 


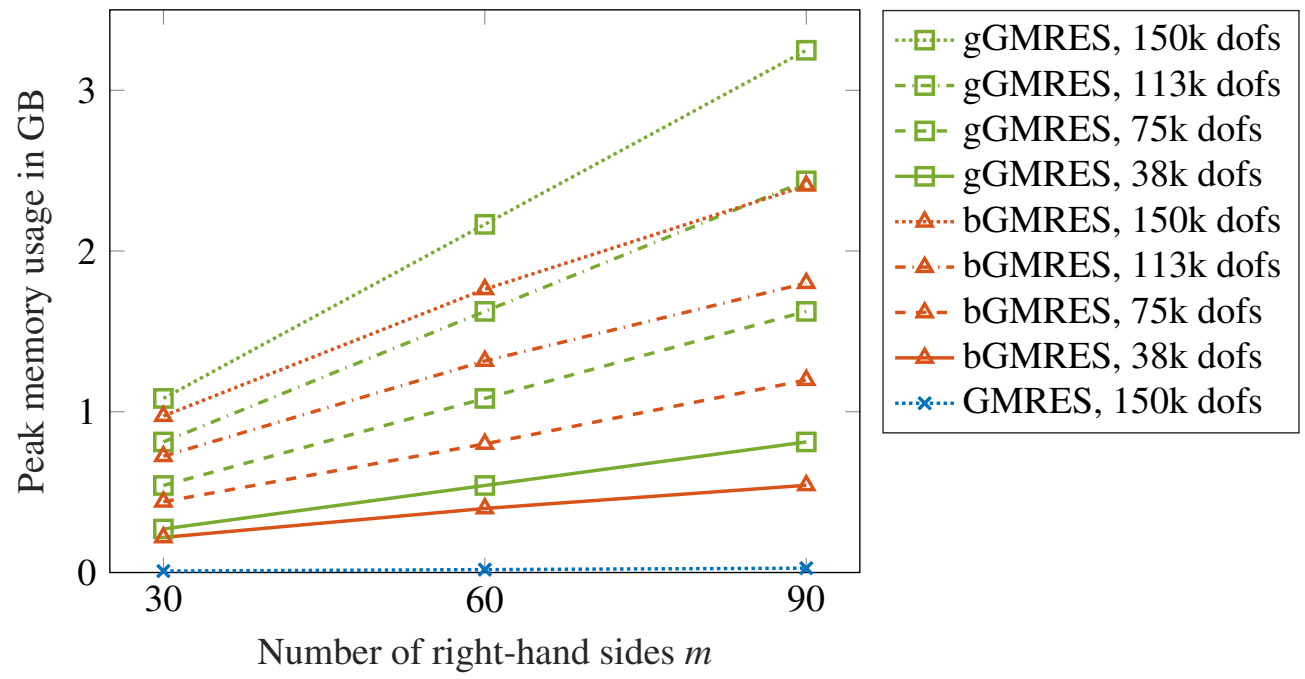

Figure 9: Peak memory usage (in gigabyte) of GMRES, global GMRES and block GMRES for solving block Toeplitz systems with 30, 60 and 90 right-hand sides considering plate models with 37600, 75200, 112800 and 150400 degrees of freedom (dofs).

between 5 and 30. This influences the accuracy of the solution and the computational effort. The accuracy of an eigenvalue $\tilde{\omega}$ and corresponding eigenvector $\boldsymbol{\Phi}$ is quantified by the relative error $|\mathbf{T}(\tilde{\omega}) \boldsymbol{\Phi}| /|\boldsymbol{\Phi}|$.

\subsubsection{Eigenvalues and eigenvectors}

Figure 10 shows the eigenvalues of the C-shaped sonic crystal within the contour. All eigenvalues have a negative imaginary part and are calculated with a relative error of less than $10^{-8}$ using $L=20$. The accuracy of the solution changes significantly with the parameter $L$ as shown in fig. 11. Each mode is numbered in ascending order according to the real part of its eigenvalue, i.e. its eigenfrequency. Figure 12 visualizes the first, third and fifth eigenvector on the boundary of the sonic crystal, with corresponding eigenfrequencies of $363.6 \mathrm{~Hz}, 426.3 \mathrm{~Hz}$ and $432.0 \mathrm{~Hz}$. Resonances within each C-shaped cylinder can be observed. In the case of the first eigenvector, the resonances of the first and fourth cylinder are in-phase and shifted by $\pi$ with respect to the resonances in the second and third cylinder. This changes for the third eigenfrequency, where the resonance of the first two cylinders and the last two cylinders share the same phase. The resonances resemble the pressure distribution of a Helmholtz resonator in the cutting planes parallel to the $x y$-plane where the sound pressure is nearly constant. The sound pressure varies along the height of the C-shaped cylinders due to the opening at the top. Three quarters of a wave forms within the resonators in the case of the fifth eigenvector with less pronounced magnitudes in the second and third cylinders. The large imaginary parts of the calculated eigenvalues indicate high attenuation of the sound waves that excite the corresponding modes. This is in good agreement with the study of Karimi et al. [13], in which significant insertion loss between $350 \mathrm{~Hz}$ to $480 \mathrm{~Hz}$ was reported for a comparable C-shaped sound barrier design.

\subsubsection{Computational effort and memory requirements}

The performance of all three Krylov solvers is evaluated and compared in terms of their solution time and peak memory usage. Figure 13(a) presents the time spent for the iterative solution of a single block Toeplitz system within the block-SS method, i.e. for computing the term $\mathbf{T}\left(z_{j}\right)^{-1} \mathbf{V}$ in eq. (29). The results are based on the solution at $z_{1}=478.56+14.63 \mathrm{i}$ but also hold for every other contour point shown 


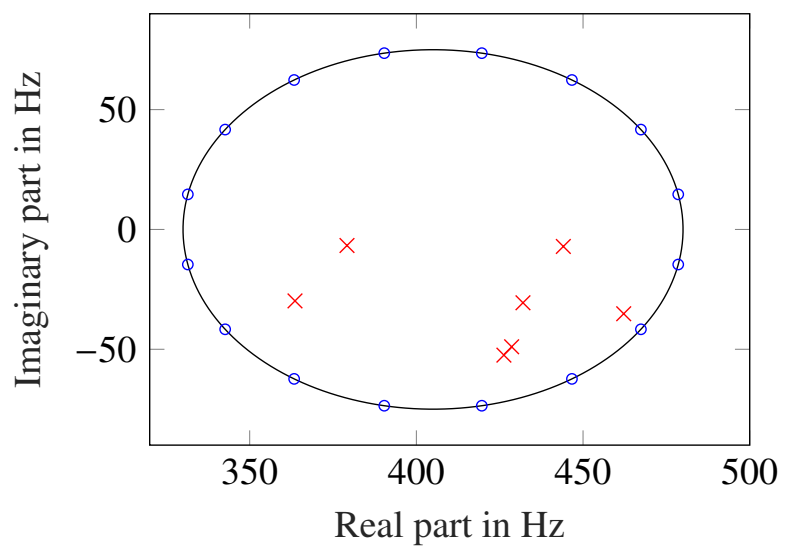

— Contour $\circ$ Contour point $\times$ Eigenvalue

Figure 10: Contour and contour points used in the block-SS method. The calculated eigenvalues lie inside the contour and are highlighted by red crosses.

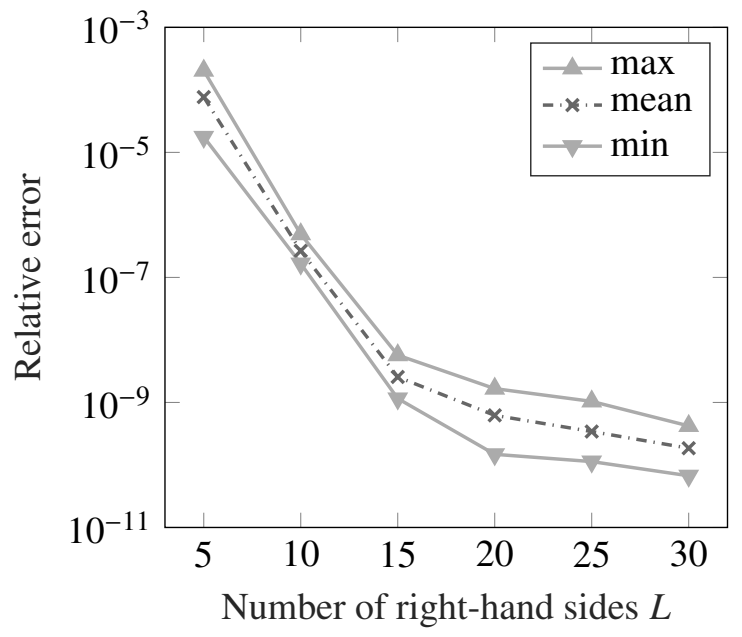

Figure 11: Convergence of the eigenvalues $\tilde{\omega}$ and eigenvectors $\boldsymbol{\Phi}$ with respect to the parameter $L$ for $N_{\mathrm{c}}=16$ contour points. The relative error is given by $|\mathbf{T}(\tilde{\omega}) \boldsymbol{\Phi}| /|\boldsymbol{\Phi}|$.

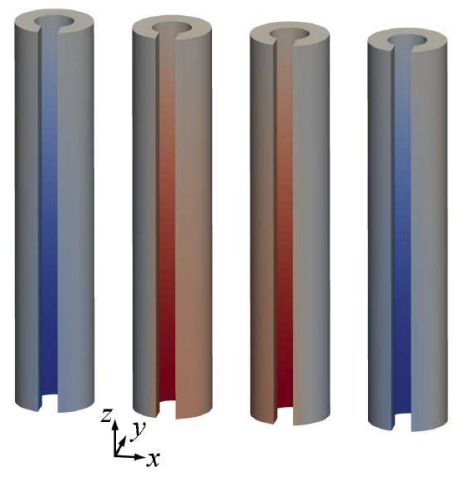

$\tilde{\omega}_{1}=363.6-29.8 \mathrm{i}$

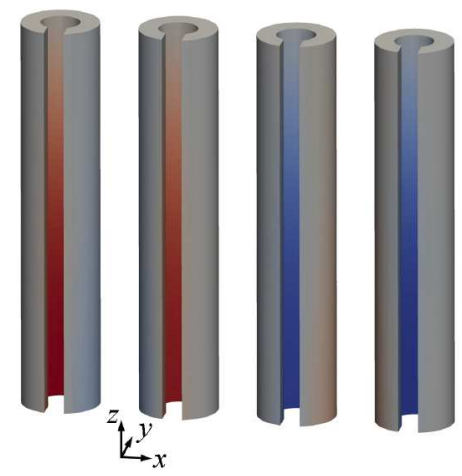

$\tilde{\omega}_{3}=426.3-52.4 \mathrm{i}$

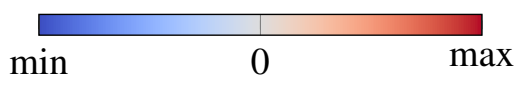

Sound pressure (real part)

Figure 12: Real part of the eigenvectors of the first, third and fifth eigenfrequency (from left to right). 


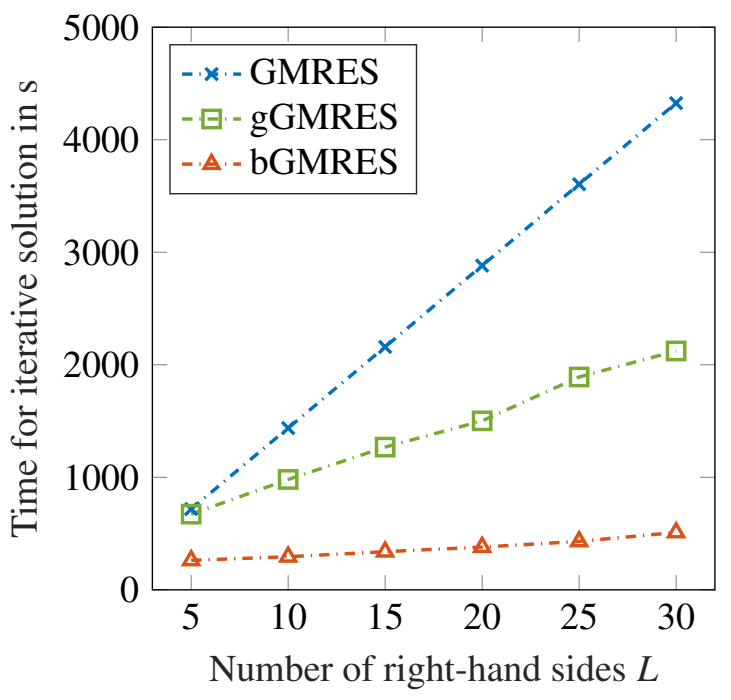

(a) Wall clock time (in seconds) for the iterative solution

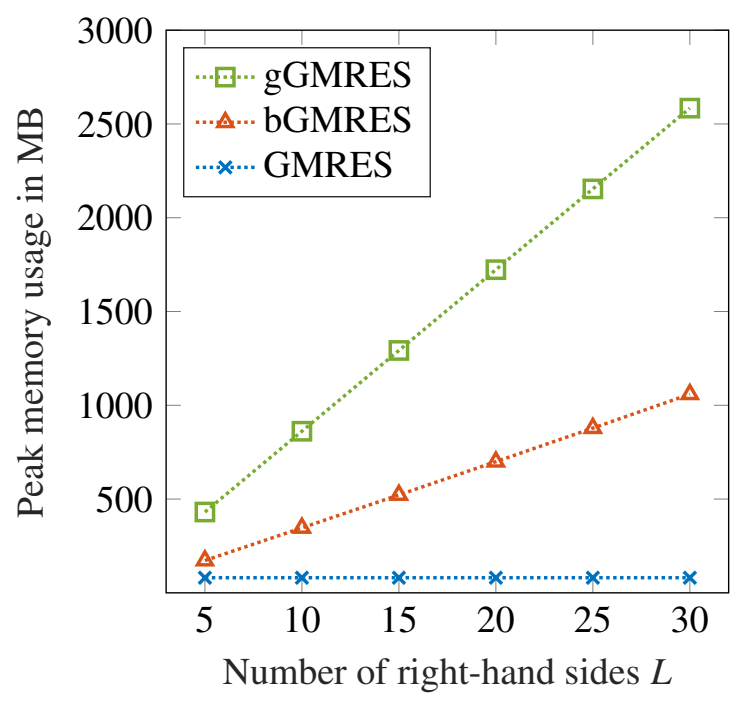

(b) Peak memory usage (in megabyte)

Figure 13: Comparison of the performance of GMRES, global GMRES (gGMRES) and block GMRES (bGMRES) based on the solution of a single sequence of block Toeplitz systems in the block-SS method. Values of $L$ between 5 and 30 are considered which leads to block Toeplitz systems with 5 to 30 right-hand sides.

in fig. 10. The value of $L$ defines the accuracy of the eigenvalue solver and corresponds to the number of columns of $\mathbf{V}$, that is, the number of right-hand sides of the block Toeplitz system. The solution time of the GMRES method scales linear with $L$ and takes $718 \mathrm{~s}$ for $L=5$ and $4325 \mathrm{~s}$ for $L=30$. An average of 106.2 iterations is performed per right-hand side. The global GMRES is up to two times faster and requires between 115 and 119 iterations in total. The number of iterations is slightly higher than in the GMRES method since the Frobenius norm of the residual matrix $\mathbf{R}$ is used to calculate the relative error of the intermediate solutions. This norm slightly overestimates the actual relative error of each individual solution vector which is given by its $l^{2}$-norm. The fastest solver is the block GMRES method which only requires 44 iterations in the case of $L=5$ and generates a solution 2.74 times faster than GMRES. The number of block GMRES iterations reduces with an increase in $L$. Only 28 iterations are performed in the case of $L=30$ which takes $510 \mathrm{~s}$ and corresponds to a speedup of 8.48 compared to GMRES and 4.16 compared to global GMRES. As shown in the previous case study, this speedup comes at the cost of higher memory requirements. Figure 13(b) compares the peak memory usage of all three solvers with respect to $L$. Since GMRES solves the block Toeplitz system for each right-hand side subsequently, the memory usage is constant in $L$ with only $80.5 \mathrm{MB}$. For $L=30$, the global and block GMRES require additional memory of up to $2584 \mathrm{MB}$ and $1059 \mathrm{MB}$, respectively. However, the additional memory usage is well within the bounds of modern desktop computers. In the case of the block variant, the speedup is in good relation with the additional memory requirements. For $L=20$, a speedup of 7.6 comes at an increase of the memory usage by 8.7 .

The convergence of the relative error within the solution process is presented in fig. 14 for all three solvers and $L=20$ right-hand sides. The GMRES method solves each block Toeplitz system subsequently without taking information of previous solution processes into account. GMRES takes a total of 2123 matrix-vector products to solve the full sequence. In contrast, the global GMRES performs matrix-matrix products and solves the system for all right-hand sides simultaneously. The matrix-matrix products are 


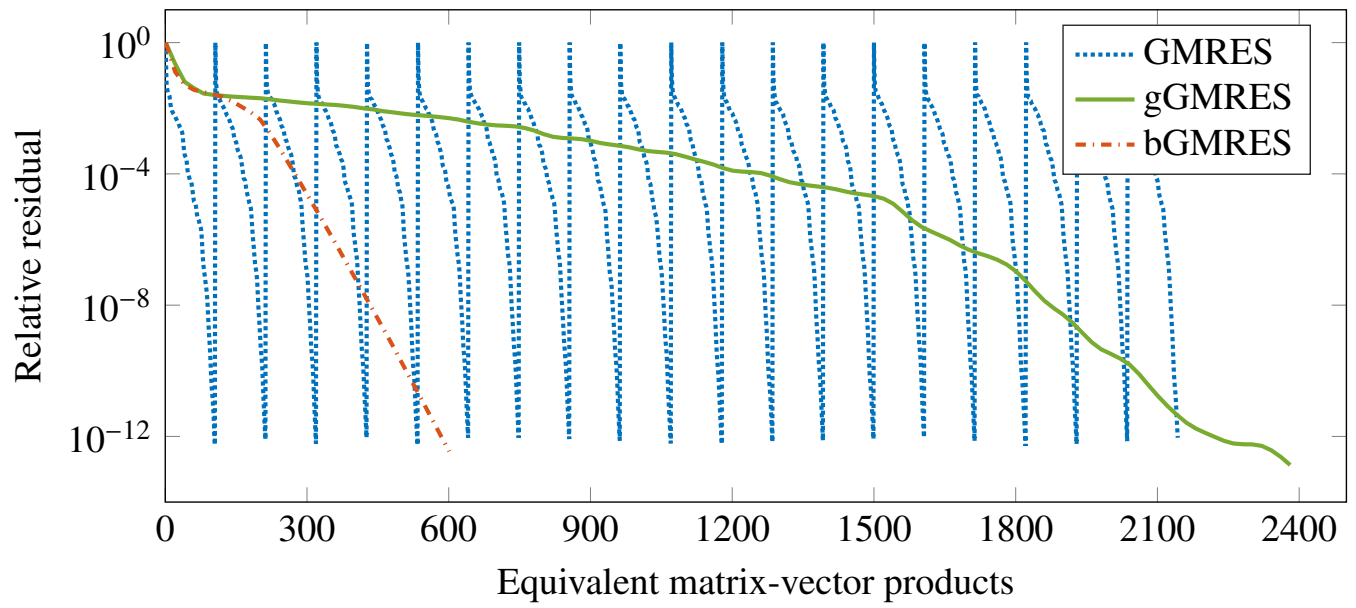

Figure 14: Convergence of the relative residual of the solutions calculated using GMRES, global GMRES (gGMRES) and block GMRES (bGMRES) for the eigenvalue analysis with $L=20$ right-hand sides. Multiplications of the system matrix with another matrix are expressed in terms of equivalent matrix-vector products.

expressed in terms of equivalent matrix-vector products in fig. 14. Applying the global GMRES method does not yield faster convergence. In fact, a few additional equivalent matrix-vector products are computed. However, significant speedup is achieved due to performing matrix-matrix products which can be computed more efficiently than matrix-vector products. The fastest convergence is achieved using the block GMRES method. It solves the full sequence at once and builds a very large Krylov subspace. In the present case, convergence is achieved in 30 iterations computing 600 equivalent matrix-vector products.

\section{Conclusions}

In this work, global and block GMRES methods were employed to solve block Toeplitz systems with many right-hand sides. Such systems can be understood as sequences of block Toeplitz systems and arise in various fields. In the present study, boundary element discretized Helmholtz problems of periodic structures are considered. Employing global and block GMRES significantly reduces the time of the iterative solution, thus providing an efficient approach for exterior acoustic problems. To demonstrate the approach, two case studies were presented. In the first case study, the evaluation of aeroacoustic noise from turbulent flow around a flat plate airfoil was examined. The block Toeplitz matrix arises from a periodic boundary element formulation applied to the geometric model divided into unit cells. The acoustic excitation was assessed by synthesizing the wall pressure field underneath the turbulent boundary layer in terms of uncorrelated wall plane waves. A number of realizations of the wall pressure field to yield a converged solution was taken into account which leads to a sequence of block Toeplitz systems. In the second case study, an eigenvalue analysis of a sonic crystal barrier was performed. Applying the periodic boundary element method yields a block Toeplitz system which is solved at various frequencies within a nonlinear eigenvalue solver in order to extract the eigenvalues and eigenvectors within a predefined contour. The block Toeplitz system features many right-hand sides and its solution accounts for the major part of the computational time spent by the nonlinear eigenvalue solver. Both numerical examples presented here show that employing global and block GMRES significantly reduces the time of the iterative solution of the sequences of block Toeplitz systems. The solution times of both methods exhibit more favorable scaling with respect to the number of righthand sides, leading to higher speedup rates for problems with an increasing number of right-hand sides. 
The speedup rates arise from performing matrix-matrix products within global and block GMRES instead of matrix-vector products with standard GMRES. A further speedup is achieved using block GMRES since the method requires less iterations. The significant reduction in solution time comes at the cost of increased memory requirements. Further acceleration of the solution process can be achieved by reducing the complexity of the matrix multiplications. In the case of aeroacoustic noise prediction, this may be done by incorporating the fast multipole BEM into the periodic boundary element formulation. For problems which feature poor convergence behavior, different preconditioning techniques based on the block Toeplitz structure of the system matrix could be applied. In addition, the block GMRES solver can be applied to other acoustic BEM problems in which many right-hand sides arise. This might include problems which do not feature block Toeplitz matrices such as the optimization of high-intensity focused ultrasonic transducer arrays $[69,70]$.

\section{Acknowledgments}

The second author gratefully acknowledges the financial support by the Australian Government through the Australian Research Councils Discovery Early Career Project funding scheme (project DE190101412).

\section{References}

[1] G. H. Golub, C. F. Van Loan, Matrix computations, 2nd ed., Johns Hopkins University Press, 1989.

[2] R. M. Gray, Toeplitz and circulant matrices: A review, Foundations and Trends in Communications and Information Theory 2 (2005) 155-239. doi:10.1561/0100000006.

[3] G. Strang, The discrete cosine transform, SIAM Review 41 (1999) 135-147. doi:10.1137/s0036144598336745.

[4] J. Haupt, W. U. Bajwa, G. Raz, R. Nowak, Toeplitz compressed sensing matrices with applications to sparse channel estimation, IEEE Transactions on Information Theory 56 (2010) 5862-5875. doi:10.1109/tit.2010.2070191.

[5] X. Antoine, C. Chniti, K. Ramdani, On the numerical approximation of high-frequency acoustic multiple scattering problems by circular cylinders, Journal of Computational Physics 227 (2008) 1754-1771. doi:10.1016/j · jcp. 2007.09.030.

[6] F. A. Amirkulova, A. N. Norris, Negative refraction of acoustic waves in phononic crystals using recursive algorithms for block Toeplitz matrices, The Journal of the Acoustical Society of America 138 (2015) 1751-1751. doi:10.1121/1.4933533.

[7] K. D. Czuprynski, J. B. Fahnline, S. M. Shontz, Parallel boundary element solutions of block circulant linear systems for acoustic radiation problems with rotationally symmetric boundary surfaces, Proceedings of Inter-Noise 2012 (2012).

[8] B. E. Barrowes, F. L. Teixeira, J. A. Kong, Fast algorithm for matrix-vector multiply of asymmetric multilevel block-Toeplitz matrices in 3-D scattering, Microwave and Optical Technology Letters 31 (2001) 28-32. doi:10.1002/mop. 1348.

[9] W.-J. Zeng, W. Xu, Fast estimation of sparse doubly spread acoustic channels, The Journal of the Acoustical Society of America 131 (2012) 303-317. doi:10.1121/1.3665992.

[10] Q. Zhang, H. Abeida, M. Xue, W. Rowe, J. Li, Fast implementation of sparse iterative covariance-based estimation for array processing, in: Conference Record of the Forty Fifth Asilomar Conference on Signals, Systems and Computers (ASILOMAR), IEEE, 2011, pp. 2031-2035. doi:10.1109/acssc.2011.6190383.

[11] H. Özbay, A. Tannenbaum, A skew Toeplitz approach to the $H^{\infty}$ optimal control of multivariable distributed systems, SIAM Journal on Control and Optimization 28 (1990) 653-670. doi:10.1137/0328038.

[12] M. Karimi, P. Croaker, N. Kessissoglou, Boundary element solution for periodic acoustic problems, Journal of Sound and Vibration 360 (2016) 129-139. doi:10.1016/j.jsv.2015.09.022.

[13] M. Karimi, P. Croaker, N. Kessissoglou, Acoustic scattering for 3D multi-directional periodic structures using the boundary element method, The Journal of the Acoustical Society of America 141 (2017) 313-323. doi:10.1121/1.4973908.

[14] M. Karimi, P. Croaker, N. Peake, N. Kessissoglou, Acoustic scattering for rotational and translational symmetric structures in nonuniform potential flow, AIAA Journal 55 (2017) 3318-3327. doi:10.2514/1. j055844.

[15] M. Karimi, P. Croaker, R. Kinns, N. Kessissoglou, Effect of a serrated trailing edge on sound radiation from nearby quadrupoles, The Journal of the Acoustical Society of America 141 (2017) 2997-3010. doi:10.1121/1.4982201.

[16] M. Karimi, P. Croaker, A. Skvortsov, D. Moreau, N. Kessissoglou, Numerical prediction of turbulent boundary layer noise from a sharp-edged flat plate, International Journal for Numerical Methods in Fluids 90 (2019) 522-543. doi:10.1002/fld. 4733. 
[17] J. Chen, T. L. H. Li, M. Anitescu, A parallel linear solver for multilevel Toeplitz systems with possibly several right-hand sides, Parallel Computing 40 (2014) 408-424. doi:10.1016/j.parco.2014.06.004.

[18] E. Bleszynski, M. Bleszynski, T. Jaroszewicz, AIM: Adaptive integral method for solving large-scale electromagnetic scattering and radiation problems, Radio Science 31 (1996) 1225-1251. doi:10.1029/96rs02504.

[19] J. Cavillot, D. Tihon, C. Craeye, E. de Lera Acedo, N. Razavi-Ghods, Fast simulation technique for antenna installed on a finite ground plane, in: 2019 International Conference on Electromagnetics in Advanced Applications (ICEAA), IEEE, 2019, pp. 0742-0745. doi:10.1109/iceaa.2019.8879358.

[20] V. Kalantzis, A. C. I. Malossi, C. Bekas, A. Curioni, E. Gallopoulos, Y. Saad, A scalable iterative dense linear system solver for multiple right-hand sides in data analytics, Parallel Computing 74 (2018) 136-153. doi:10.1016/j.parco.2017.12. 005.

[21] N. Levinson, The Wiener RMS error criterion in filter design and prediction, Journal of Mathematics and Physics 25 (1946) 261-278. doi:10.1002/sapm1946251261.

[22] W. F. Trench, Solution of systems with Toeplitz matrices generated by rational functions, Linear Algebra and its Applications 74 (1986) 191-211. doi:10.1016/0024-3795 (86)90122-9.

[23] I. Gohberg, T. Kailath, I. Koltracht, Efficient solution of linear systems of equations with recursive structure, Linear Algebra and its Applications 80 (1986) 81-113. doi:10.1016/0024-3795(86)90279-x.

[24] N. Kalouptsidis, G. Carayannis, D. Manolakis, Fast algorithms for block Toeplitz matrices with Toeplitz entries, Signal Processing 6 (1984) 77-81. doi:10.1016/0165-1684(84)90053-7.

[25] S. Chandrasekaran, A. H. Sayed, A fast stable solver for nonsymmetric Toeplitz and quasi-Toeplitz systems of linear equations, SIAM Journal on Matrix Analysis and Applications 19 (1998) 107-139. doi:10.1137/s0895479895296458.

[26] R. P. Brent, F. G. Gustavson, D. Y. Y. Yun, Fast solution of Toeplitz systems of equations and computation of Padé approximants, Journal of Algorithms 1 (1980) 259-295. doi:10.1016/0196-6774 (80)90013-9.

[27] S. Chandrasekaran, M. Gu, X. Sun, J. Xia, J. Zhu, A superfast algorithm for Toeplitz systems of linear equations, SIAM Journal on Matrix Analysis and Applications 29 (2008) 1247-1266. doi:10.1137/040617200.

[28] P. G. Martinsson, V. Rokhlin, M. Tygert, A fast algorithm for the inversion of general Toeplitz matrices, Computers \& Mathematics with Applications 50 (2005) 741-752. doi:10.1016/j . camwa .2005.03.011.

[29] M. Stewart, A superfast Toeplitz solver with improved numerical stability, SIAM Journal on Matrix Analysis and Applications 25 (2003) 669-693. doi:10.1137/s089547980241791x.

[30] R. H.-F. Chan, X.-Q. Jin, An introduction to iterative Toeplitz solvers, Society for Industrial and Applied Mathematics, 2007. doi:10.1137/1.9780898718850.

[31] G. Strang, A proposal for Toeplitz matrix calculations, Studies in Applied Mathematics 74 (1986) 171-176. doi:10.1002/ sapm1986742171.

[32] X.-Q. Jin, Developments and applications of block Toeplitz iterative solvers, Springer Netherlands, 2003.

[33] R. H. Chan, P. T. P. Tang, Fast band-Toeplitz preconditioners for Hermitian Toeplitz systems, SIAM Journal on Scientific Computing 15 (1994) 164-171. doi:10.1137/0915011.

[34] D. Bini, F. Benedetto, A new preconditioner for the parallel solution of positive definite Toeplitz systems, in: Proceedings of the second annual ACM symposium on Parallel algorithms and architectures - SPAA '90, ACM Press, 1990, pp. $220-223$. doi:10.1145/97444.97688.

[35] J. Chen, T. L. H. Li, Parallelizing the conjugate gradient algorithm for multilevel Toeplitz systems, Procedia Computer Science 18 (2013) 571-580. doi:10.1016/j.procs .2013.05.221.

[36] Y. Saad, M. H. Schultz, GMRES: a generalized minimal residual algorithm for solving nonsymmetric linear systems, SIAM Journal on Scientific and Statistical Computing 7 (1986) 856-869. doi:10.1137/0907058.

[37] Y. Saad, Iterative Methods for Sparse Linear Systems, Society for Industrial and Applied Mathematics, 2003. doi:10 .1137/ 1.9780898718003.

[38] M. H. Gutknecht, Block Krylov space methods for linear systems with multiple right-hand sides: An introduction, in: A. H. Siddiqi, I. Duff, O. Christensen (Eds.), Modern Mathematical Models, Methods and Algorithms for Real World Systems, Anamaya Publishers, 2007, pp. 1-22.

[39] T. F. Chan, W. L. Wan, Analysis of projection methods for solving linear systems with multiple right-hand sides, SIAM Journal on Scientific Computing 18 (1997) 1698-1721. doi:10.1137/s1064827594273067.

[40] M. L. Parks, E. de Sturler, G. Mackey, D. D. Johnson, S. Maiti, Recycling Krylov subspaces for sequences of linear systems, SIAM Journal on Scientific Computing 28 (2006) 1651-1674. doi:10.1137/040607277.

[41] A. Gaul, Recycling Krylov subspace methods for sequences of linear systems, Ph.D. thesis, TU Berlin, 2014. doi:10 .14279/ depositonce-4147.

[42] H. C. Elman, O. G. Ernst, D. P. O'Leary, M. Stewart, Efficient iterative algorithms for the stochastic finite element method with application to acoustic scattering, Computer Methods in Applied Mechanics and Engineering 194 (2005) 1037-1055. doi:10.1016/j . cma. 2004.06.028. 
[43] J. Langou, Iterative methods for solving linear systems with multiple right-hand sides, Ph.D. thesis, L'Institut National des Sciences Appliques de Toulouse, 2003.

[44] L. Giraud, S. Gratton, E. Martin, Incremental spectral preconditioners for sequences of linear systems, Applied Numerical Mathematics 57 (2007) 1164-1180. doi:10.1016/j . apnum.2007.01.005.

[45] A. El Guennouni, K. Jbilou, H. Sadok, A block version of BiCGSTAB for linear systems with multiple right-hand sides, Electronic Transactions on Numerical Analysis 16 (2003) 2.

[46] V. Puzyrev, J. M. Cela, A review of block Krylov subspace methods for multisource electromagnetic modelling, Geophysical Journal International 202 (2015) 1241-1252. doi:10.1093/gji/ggv216.

[47] M. Malhotra, R. W. Freund, P. M. Pinsky, Iterative solution of multiple radiation and scattering problems in structural acoustics using a block quasi-minimal residual algorithm, Computer Methods in Applied Mechanics and Engineering 146 (1997) 173-196. doi:10.1016/s0045-7825 (96)01227-3.

[48] H. Calandra, S. Gratton, R. Lago, X. Vasseur, L. M. Carvalho, A modified block flexible GMRES method with deflation at each iteration for the solution of non-Hermitian linear systems with multiple right-hand sides, SIAM Journal on Scientific Computing 35 (2013) S345-S367. doi:10.1137/120883037.

[49] J. Asakura, T. Sakurai, H. Tadano, T. Ikegami, K. Kimura, A numerical method for nonlinear eigenvalue problems using contour integrals, JSIAM Letters 1 (2009) 52-55. doi:10.14495/jsiaml.1.52.

[50] W.-J. Beyn, An integral method for solving nonlinear eigenvalue problems, Linear Algebra and its Applications 436 (2012) 3839-3863. doi:10.1016/j.laa.2011.03.030.

[51] B. Gavin, A. Międlar, E. Polizzi, FEAST eigensolver for nonlinear eigenvalue problems, Journal of Computational Science 27 (2018) 107-117. doi:10.1016/j.jocs . 2018.05.006.

[52] P. Davis, Circulant Matrices, AMS Chelsea Publishing Series, Chelsea, New York, 1994.

[53] B. Vital, Etude de quelques méthodes de résolution de problemes linéaires de grande taille sur multiprocesseur, Ph.D. thesis, Université de Rennes, 1990.

[54] R. B. Morgan, GMRES with deflated restarting, SIAM Journal on Scientific Computing 24 (2002) 20-37. doi:10.1137/ s1064827599364659.

[55] A. H. Baker, J. M. Dennis, E. R. Jessup, On improving linear solver performance: A block variant of GMRES, SIAM Journal on Scientific Computing 27 (2006) 1608-1626. doi:10.1137/040608088.

[56] K. Jbilou, A. Messaoudi, H. Sadok, Global FOM and GMRES algorithms for matrix equations, Applied Numerical Mathematics 31 (1999) 49-63. doi:10.1016/s0168-9274(98)00094-4.

[57] L. Maxit, Simulation of the pressure field beneath a turbulent boundary layer using realizations of uncorrelated wall plane waves, The Journal of the Acoustical Society of America 140 (2016) 1268-1285. doi:10.1121/1.4960516.

[58] M. Karimi, P. Croaker, L. Maxit, O. Robin, A. Skvortsov, S. Marburg, N. Kessissoglou, A hybrid numerical approach to predict the vibrational responses of panels excited by a turbulent boundary layer, Journal of Fluids and Structures 92 (2020) 102814. doi:10.1016/j.jfluidstructs.2019.102814.

[59] W. R. Graham, A comparison of models for the wavenumber-frequency spectrum of turbulent boundary layer pressures, Journal of Sound and Vibration 206 (1997) 541-565. doi:10.1006/jsvi.1997.1114.

[60] S. Marburg, Boundary element method for time-harmonic acoustic problems, in: M. Kaltenbacher (Ed.), Computational Acoustics, Springer, Wien, 2018.

[61] R. Martínez-Sala, J. Sancho, J. V. Sánchez, V. Gómez, J. Llinares, F. Meseguer, Sound attenuation by sculpture, Nature 378 (1995) 241-241. doi:10.1038/378241a0.

[62] L. Fredianelli, A. D. Pizzo, G. Licitra, Recent developments in sonic crystals as barriers for road traffic noise mitigation, Environments 6 (2019) 14. doi:10.3390/environments6020014.

[63] F. Montiel, H. Chung, M. Karimi, N. Kessissoglou, An analytical and numerical investigation of acoustic attenuation by a finite sonic crystal, Wave Motion 70 (2017) 135-151. doi:10.1016/j . wavemoti.2016.12.002.

[64] A. Melnikov, M. Maeder, N. Friedrich, Y. Pozhanka, A. Wollmann, M. Scheffler, S. Oberst, D. Powell, S. Marburg, Acoustic metamaterial capsule for reduction of stage machinery noise, The Journal of the Acoustical Society of America 147 (2020) 1491-1503. doi:10.1121/10.0000857.

[65] D. P. Elford, L. Chalmers, F. V. Kusmartsev, G. M. Swallowe, Matryoshka locally resonant sonic crystal, The Journal of the Acoustical Society of America 130 (2011) 2746-2755. doi:10.1121/1.3643818.

[66] L. Moheit, S. Anthis, J. Heinz, F. Kronowetter, S. Marburg, Analysis of scattering by finite sonic crystals in free field with infinite elements and normal modes, Journal of Sound and Vibration 476 (2020) 115291. doi:10.1016/j.jsv. 2020. 115291.

[67] C.-J. Zheng, H.-F. Gao, L. Du, H.-B. Chen, C. Zhang, An accurate and efficient acoustic eigensolver based on a fast multipole BEM and a contour integral method, Journal of Computational Physics 305 (2016) 677-699. doi:10.1016/j.jcp. 2015. 10.048.

[68] S. Yokota, T. Sakurai, A projection method for nonlinear eigenvalue problems using contour integrals, JSIAM Letters 5 
(2013) 41-44. doi:10.14495/jsiaml .5.41.

[69] L. R. Gavrilov, J. W. Hand, A theoretical assessment of the relative performance of spherical phased arrays for ultrasound surgery, IEEE Transactions on Ultrasonics, Ferroelectrics and Frequency Control 47 (2000) 125-139. doi:10.1109/58. 818755.

[70] E. van't Wout, P. Gélat, T. Betcke, S. Arridge, A fast boundary element method for the scattering analysis of high-intensity focused ultrasound, The Journal of the Acoustical Society of America 138 (2015) 2726-2737. doi:10.1121/1.4932166. 